\title{
Forces and frequency shifts in atomic-resolution dynamic-force microscopy
}

\author{
Franz J. Giessibl ${ }^{*}$ \\ Universität Augsburg, Experimentalphysik VI-EKM, Universitätsstrasse 1, D-86135 Augsburg, Germany
}

(Received 8 July 1997)

\begin{abstract}
True atomic resolution in vacuum with a force microscope is now obtained routinely by using the frequency shift of an oscillating cantilever as the imaging signal. Here, a calculation is presented that relates the frequency shift to the forces between tip and sample for both large and small oscillation amplitudes. Also, the frequency versus distance data for van der Waals dominated tip-sample interactions is related to the geometry of the tip apex. Published frequency versus distance data are used to show that the apex of tips providing atomic resolution is faceted and not rounded. Further, an extended jump-to-contact criterion for large amplitudes is established. [S0163-1829(97)00748-0]
\end{abstract}

\section{INTRODUCTION}

The invention of the atomic-force microscope ${ }^{1}$ (AFM) in 1985 has raised the hope to extend the spectacular resolution capability of the scanning tunneling microscope (STM) to nonconducting surfaces. However, obtaining true atomic resolution by AFM is much more difficult than by STM mainly for two reasons:

(a) In STM, the imaging signal is a tunneling current $I_{t}$ $\left(I_{t} \approx 0.1 \mathrm{nA}\right)$ that can be easily converted into a signal voltage for further processing (a simple current/voltage converter can be built with an operational amplifier and a feedback resistor). In AFM, the imaging signal is the force $F_{t s}$ between a sharp tip and a surface $\left(F_{t s} \approx 0.1 \mathrm{nN}\right)$. The conversion of $F_{t s}$ into an electrical signal is usually done by measuring the deflection of a cantilever beam (CL). Because this measurement process is indirect, it is more difficult and more prone to experimental drift and error.

(b) Atomic resolution is possible if the contribution of the foremost tip atom ("front atom") relative to rest of the tip atoms to the total imaging signal is significant. $I_{t}$ between two metal electrodes in vacuum increases by a factor of approximately 10 when decreasing the distance between the electrodes by $0.1 \mathrm{~nm}$. Thus, even for a relatively blunt tip (with a radius of say $100 \mathrm{~nm}$ ) the likelihood is high that the front atom protrudes far enough out of the tip to carry the major part of the tunneling current. $F_{t s}$ varies much less with distance, especially the van der Waals ( $\mathrm{vdW}$ ) forces have a long range and a large magnitude, ${ }^{2}$ therefore AFM tips need to be extremely sharp for producing true atomic resolution.

Problem (a) has been solved by frequency modulationAFM (FM-AFM). ${ }^{3}$ In this technique, a CL is oscillating with a constant amplitude $A_{0}$ at frequency $f .^{5} f$ depends on the eigenfrequency $f_{0}$ of the $\mathrm{CL}$ (determined by spring constant $k$ and effective mass $m^{*}$ ) and $F_{t s}$. An image is created by scanning in the $x y$ plane and keeping $f$ constant by controlling $z$. Problem (b) is solved by using extremely sharp tips, which are now available commercially. ${ }^{6}$

FM-AFM with large amplitudes has made it possible recently to achieve atomic resolution of even reactive surfaces in ultrahigh vacuum. The silicon $(111)-(7 \times 7)$ reconstruction, ${ }^{7-12}$ defects on InP, ${ }^{13}$ and insulating surfaces ${ }^{14,15}$ have been imaged by FM-AFM. It has been found that $A_{0}$ has to be very large compared to the range of $F_{t s}$ for obtaining optimal resolution, therefore the connection between $f$ and $F_{t s}$ is complicated. In this paper, $f$ is calculated as a function of distance with a simple model for $F_{t s}$. Comparison with published experimental results confirms both the results of the calculation and the validity of the simple tip model.

\section{CALCULATION OF FREQUENCY SHIFT VERSUS DISTANCE}

The motion of the CL can be described by a weakly disturbed harmonic oscillator. Figure 1 shows the deflection $q^{\prime}(t)$ of the tip of the CL: it oscillates with an amplitude $A_{0}$ at a distance $q(t)$ to a sample. The closest point to the sample is $q=d$ and $q(t)=q^{\prime}(t)+d+A_{0}$. The unperturbed Hamiltonian of the CL is

$$
H_{0}=\frac{p^{2}}{2 m^{*}}+\frac{k q^{\prime 2}}{2},
$$

where $p=m^{*} d q^{\prime} / d t$. The unperturbed motion is given by

$$
q^{\prime}(t)=A_{0} \cos \left(2 \pi f_{0} t\right)
$$

and the frequency is

$$
f_{0}=\frac{1}{2 \pi} \sqrt{\frac{k}{m^{*}}} \text { with period } T_{0}=\frac{1}{f_{0}} .
$$

The tip of the CL interacts with a surface. In many cases, forces between tip and sample can be well described by an inverse power law

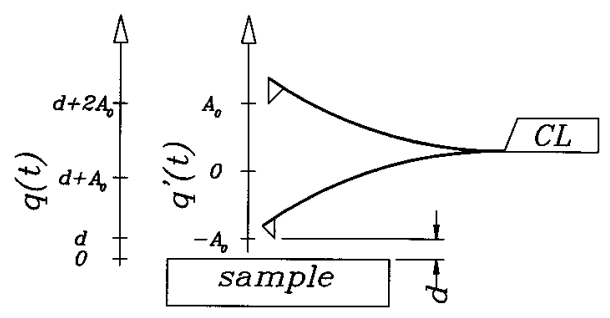

FIG. 1. Schematic of the cantilever next to a sample and definition of $q$ and $q^{\prime}$. 


$$
F_{t s}(q)=-C q^{-n}
$$

with a tip-sample force constant $C$, resulting in a perturbation Hamiltonian:

$$
\begin{gathered}
\Delta H=V_{t s}(q)=-\frac{C}{(n-1) q^{n-1}} \text { for } n>1, \\
V_{t s}(q)=-C \ln (q) \text { for } n=1 .
\end{gathered}
$$

Here, the Hamilton-Jacobi formalism ${ }^{16}$ is used to derive an analytical expression for $f$ as a function of $F_{t s}$. The momentum $p$ and deflection $q^{\prime}$ are transformed to action $(J)$-and angle $(\beta)$-variables:

$$
\begin{aligned}
& q^{\prime}=\sqrt{\frac{2 f_{0} J}{k}} \sin 2 \pi\left(f_{0} t+\beta\right), \\
& p=\sqrt{\frac{k J}{2 \pi^{2} f_{0}}} \cos 2 \pi\left(f_{0} t+\beta\right) .
\end{aligned}
$$

Comparison of Eqs. (2) and (6) yields $J=k A_{0}^{2} / 2 f_{0}$ and $\beta$ $=1 / 4$ for the unperturbed case $\left(V_{t s} \equiv 0\right)$. If $V_{t s} \neq 0, J$ and $\beta$ vary with time according to

$$
\frac{d J}{d t}=-\frac{\partial H}{\partial \beta}, \quad \frac{d \beta}{d t}=\frac{\partial H}{\partial J} .
$$

If $\max \left[\left|\partial \Delta H\left(q^{\prime}\right) / \partial q^{\prime}\right|\right] \ll \max \left[\left|\partial H_{0}\left(q^{\prime}\right) / \partial q^{\prime}\right|\right]$ for $-A_{0} \leqslant q^{\prime}$ $\leqslant A_{0}$, i.e., if $\left|F_{t s}\left(q^{\prime}=-A_{0}\right)\right|=|C| d^{-n} \ll k A_{0}$ the motion is still approximately harmonic with a modified frequency $f$ $=f_{0}+\Delta f$ and $\partial H / \partial J \approx \partial V_{t s} / \partial J$. The frequency shift is

$$
\Delta f=\left\langle\frac{d \beta}{d t}\right\rangle=\left\langle\frac{\partial V_{t s}}{\partial J}\right\rangle=\left\langle\frac{\partial V_{t s}}{\partial q^{\prime}} \frac{\partial q^{\prime}}{\partial J}\right\rangle .
$$

Differentiation of Eq. (6) yields $\partial q^{\prime} / \partial J=q^{\prime} / 2 J$ $=\left(f_{0} / k A_{0}^{2}\right) q^{\prime}$ and

$$
\Delta f=-\frac{f_{0}}{k A_{0}^{2}}\left\langle F_{t s} q^{\prime}\right\rangle .
$$

The average has to be taken for a full cycle. Inserting Eqs. (2) and (4) into Eq. (10) yields

$$
\begin{aligned}
\Delta f & \left(d, k, A_{0}, f_{0}, n\right) \\
& =\frac{f_{0}}{k A_{0}^{2}} \frac{1}{T_{0}} \int_{0}^{T_{0}} \frac{C A_{0} \cos \left(2 \pi f_{0} t\right)}{\left\{d+A_{0}+A_{0}\left[\cos \left(2 \pi f_{0} t\right)+1\right]\right\}^{n}} d t \\
& =\frac{1}{2 \pi} \frac{f_{0}}{k A_{0}} \frac{C}{d^{n}} \int_{0}^{2 \pi} \frac{\cos x}{\left[1+\left(A_{0} / d\right)(\cos x+1)\right]^{n}} d x .
\end{aligned}
$$

This expression can be simplified for two limiting cases:

\section{A. Small amplitudes $A_{\mathbf{0}} \ll d$}

Making use of the identities $(1+\epsilon)^{-n} \approx 1-n \epsilon$ for $\epsilon \ll 1$ and $\int_{0}^{2 \pi} \cos ^{2} x d x=\pi$ yields

$$
\Delta f\left(d, k, f_{0}, n\right) \cong-n \frac{f_{0}}{2 k} \frac{C}{d^{n+1}}=\frac{f_{0}}{2 k} \frac{\partial^{2} V_{t s}(q=d)}{\partial q^{2}} .
$$

This corresponds to the expression $f=(1 / 2 \pi) \sqrt{k_{\text {eff }} / m}$ with $k_{\text {eff }}=k+\partial^{2} V_{t s} / \partial q^{2}$, given in the initial work by Albrecht et $a l^{3}$

\section{B. Large amplitudes $A_{0} \gg d$}

Taylor series expansion of the denominator in Eq. (11) around $x_{0}=\pi\left(x^{\prime}=x-\pi, \cos x \approx-1+x^{\prime 2} / 2\right)$ and substitution $\left(y=\sqrt{A_{0} / 2 d} x^{\prime}\right)$ yields

$$
\Delta f\left(d, k, A_{0}, f_{0}, n\right) \cong-\frac{1}{\sqrt{2} \pi} \frac{f_{0}}{k A_{0}^{3 / 2}} \frac{C}{d^{n-1 / 2}} I_{1}(n),
$$

with

$$
I_{1}(n):=\int_{-\infty}^{\infty} \frac{1}{\left(1+y^{2}\right)^{n}} d y .
$$

Pertinent values of $I_{1}(n)$ are $I_{1}(1)=\pi, I_{1}(2) \approx 1.6, I_{1}(3)$ $\approx 1.2, I_{1}(4) \approx 0.98, I_{1}(7) \approx 0.7$, and $I_{1}(13) \approx 0.5$.

Since $\Delta f \propto f_{0} / k A_{0}^{3 / 2}$ for large amplitudes for all inverse power forces and even for exponential ${ }^{17}$ tip-sample forces, independent of the exponent $n$, the set of four parameters $\left(\Delta f, k, A_{0}, f_{0}\right)$ that is currently needed for a full description of an FM-AFM experiment can be condensed in a single parameter, a "normalized frequency shift":

$$
\gamma(d):=\frac{\Delta f\left(d, k, A_{0}, f_{0}\right) k A_{0}^{3 / 2}}{f_{0}} .
$$

Since $\Delta f$ is linear in $F_{t s}$ [Eq. (10)], $\gamma$ is also linear and

$$
\gamma(d) \cong-\frac{1}{\sqrt{2} \pi} \sum_{n=1}^{\infty} \frac{C_{n} I_{1}(n)}{d^{n-1 / 2}}
$$

if $F_{t s}$ can be expanded in a Laurent series $F_{t s}(q)=$ $-\sum_{n=1}^{\infty} C_{n} / q^{n}$.

\section{CALCULATION OF TIP-SAMPLE FORCES}

The theory of forces between tip and sample in AFM is the subject of several overviews ${ }^{18}$ and the case of a Si tip and a Si $(111)-(5 \times 5)$ sample has been treated in detail by Perez et al. ${ }^{19}$ Here, a simple model is used that can easily be adapted to the experimental conditions.

\section{A. Distances greater than interatomic spacings}

The nonretarded ( $\mathrm{vdW}$ ) energy in vacuum for two atoms at distance $q$ is ${ }^{20}$

$$
V_{\text {atom-atom }}(q)=-\frac{C_{\mathrm{vdW}}}{q^{6}},
$$

where $C_{\mathrm{vdW}}$ is the atomic vdW interaction constant. Assuming additivity, this equation can be used to calculate the $\mathrm{vdW}$ interaction for macroscopic bodies, specifically an AFM tip and a flat sample. If the flat sample (surface in $x y$ plane) is modeled by a continuum with an atom density $\rho_{s}$ for $z \leqslant 0$, the vdW energy of a single atom at height $z=q$ can be calculated from Eq. (17) by a so-called Hamaker integration 
(i.e., the summation over all sample atoms is replaced by an integration over the sample volume times the atom density of the sample material):

$$
V_{\text {atom-sample }}(q)=-\frac{\pi C_{\mathrm{vdW}} \rho_{s}}{q^{3}} .
$$

A second Hamaker integration over the tip volume (atom density $\rho_{t}$ ) yields the total vdW potential for the tip. Without loss of generality, the tip geometry can be defined by its cross section (in the $x y$ plane) $A(h)$ at height $h$. If the front atom of this tip is at distance $z=q$, its $\mathrm{vdW}$ energy is

$$
V_{t s, \mathrm{vdW}}(q)=-\frac{\pi C_{\mathrm{vdW}} \rho_{s} \rho_{t}}{6} \int_{h=0}^{H} \frac{A(h)}{(q+h)^{3}} d h .
$$

The quantity $\pi^{2} C_{\mathrm{vdw}} \rho_{t} \rho_{s}$ is usually referred to as the Hamaker constant $A_{H}$, and $A_{H} \approx 0.1 \mathrm{aJ}$ for most condensed phases and interactions across vacuum. ${ }^{20}$ If $A(h)=\chi h^{m}$, then the set of $m$ and $\chi$ for various types of tips is

\begin{tabular}{llll}
\multicolumn{1}{c}{ Type } & $m$ & \multicolumn{1}{c}{$\chi$} & \\
\hline pyramidal & 2 & $4 \tan ^{2}(\alpha / 2)$ & $\alpha$ full tip angle \\
conical & 2 & $\pi \tan ^{2}(\alpha / 2)$ & $R:$ radius of tip apex \\
parabolic & 1 & $2 \pi R$ & $w:$ width of wedge \\
wedge-type & 1 & $2 \tan (\alpha / 2) w$ & $A:$ cross section of rod (constant) \\
rod-type & 0 & $A$ &
\end{tabular}

With this set, a variety of tips can be modeled, including crushed tips. $^{21}$

For $m=2$ :

$$
\begin{aligned}
V_{\mathrm{vdW}, m=2}(q)= & -A_{H} \frac{\chi}{6 \pi}\left\{\ln (1+H / q)+\frac{2}{1+H / q}\right. \\
& \left.-\frac{1}{2(1+H / q)^{2}}-\frac{3}{2}\right\},
\end{aligned}
$$

where $H$ is the total height. $V_{\mathrm{vdW}, m=2}$ diverges for $H \rightarrow \infty$, but the force $F_{\mathrm{vdW}, m=2}(q)=-\partial V_{\mathrm{vdW}, m=2}(q) / \partial q$ is finite:

$$
\begin{aligned}
F_{\mathrm{vdW}, m=2}(q) & =-A_{H} \frac{\chi}{6 \pi} \frac{1}{q(1+q / H)^{3}} \\
& \rightarrow-A_{H} \frac{\chi}{6 \pi} \frac{1}{q} \text { for } H \rightarrow \infty .
\end{aligned}
$$

Thus, for infinitely high parabolic and conical tips the contribution of the front section from $h=0$ to $h=n q$ to the total $F_{t s}$ is $(1+1 / n)^{-3}$. For a tip at distance $q=1 \mathrm{~nm}$ the section between $h=0$ and $h=10 \mathrm{~nm}$ gives rise to $75 \%$ of the total $F_{t s} . F_{t s}(q)$ for $0<q<1 \mathrm{~nm}$ is only dependent on the geometry of the first $10 \mathrm{~nm}$ of the front section, i.e., if $\alpha=130^{\circ}$ for the first $10 \mathrm{~nm}$ and then decreases to $30^{\circ}$ for the rest of the tip, this shift in angle is barely noticeable in $F_{t s}(q)$ for $0<q<1 \mathrm{~nm}$.

For infinitely high tips with $m<2, V_{\mathrm{vdW}}(q)$ is given by

$$
V_{\mathrm{vdW}}(q)=-A_{H} \frac{\chi}{6 \pi} I_{2}(m) \frac{1}{q^{2-m}}
$$

with $I_{2}(m):=\int_{0}^{\infty}\left[y^{m} /(1+y)^{3}\right] d y$ and $I_{2}(0)=I_{2}(1)=0.5$.

Equations (22) and (13) have a very interesting implication: if $F_{t s}$ is dominated by vdW forces and the tip can be described by $A(h)=\chi h^{m}$ then

$$
\Delta f(d) \propto d^{-2.5+m} .
$$

A plot of $-\ln (-\Delta f)$ versus $\ln (d)$ is a straight line with a slope $2.5-m$, parabolic, spherical, and wedge-type tips produce a slope of 1.5 , pyramidal and conical tips produce a slope of 0.5 .

\section{B. Distances in the order of interatomic spacings}

Approximating tip and sample by a continuum is valid if $q$ is significantly greater than the interatomic distances. If $q$ is in the order of the next-neighbor distance $\sigma$, the "continuum," vdW force can be superseded by the vdW force between the front atom and the sample atom closest to it. If tip and sample have exposed dangling bonds, chemical bonding can also occur. For simplicity, this bond is modeled by a Lennard-Jones (LJ) potential with bond energy $E_{\text {bond }}$ and equilibrium distance $\sigma$ :

$$
V_{\mathrm{LJ}}(q)=-E_{\text {bond }}\left[2\left(\frac{\sigma}{q}\right)^{6}-\left(\frac{\sigma}{q}\right)^{12}\right] .
$$

The force is given by

$$
F_{\mathrm{LJ}}(q)=-12 \frac{E_{\text {bond }}}{\sigma}\left[\left(\frac{\sigma}{q}\right)^{7}-\left(\frac{\sigma}{q}\right)^{13}\right] .
$$

The pair potential can now be calculated from the bulk silicon properties. Silicon has a binding energy of $4.63 \mathrm{eV}$ per atom. ${ }^{22}$ Assuming that this binding energy is due to the nextneighbor covalent bonds, the energy of one bond is $E_{\text {bond }}=2.315 \mathrm{eV}=0.371 \mathrm{aJ}$. The nearest-neighbor distance is $\sigma=(\sqrt{3} / 4) a_{0}=0.235 \mathrm{~nm}$.

Stillinger and Weber have calculated a more sophisticated potential for two silicon atoms. ${ }^{23}$ However, the deviations of the simple LJ potential are insignificant for the purpose here, the maximal attractive force according to the SW potential is $F_{\mathrm{SW}}^{\max }\left(q^{*}\right) \approx-5 \mathrm{nN}$ with $q^{*}=0.27 \mathrm{~nm}$, whereas $F_{\mathrm{LJ}}^{\max }\left(q^{*}\right) \approx$ $-4.25 \mathrm{nN}$ with $q^{*}=0.255 \mathrm{~nm}$. 


\section{Total tip-sample force}

With the results above, the total $F_{t s}$ for an idealized pyramidal silicon tip that is bounded by (111) planes (full tip angle $\alpha=70.5^{\circ}, A_{H}=0.1865 \mathrm{aJ}^{19}$ ) at a distance $q$ to a flat silicon surface is calculated explicitly. It is instructive to express $F_{t s}$ as a function of $q / \sigma$ :

$$
F_{t s}(q)=-0.1 \mathrm{nN} \frac{\sigma}{q}-18.9 \mathrm{nN}\left(\frac{\sigma^{7}}{q^{7}}-\frac{\sigma^{13}}{q^{13}}\right)
$$

For distances $q \geqslant 2 \sigma, F_{t s}$ is dominated by the vdW contribution. For $q^{*} \lesssim q \lesssim 2 \sigma, F_{t s}$ is mainly due to the LJ forces, with a slowly varying "continuum vdW' contribution. However, as noted above, the continuum vdW calculation is not valid for very small distances, in fact the vdW force between the front atom and the sample atom closest to it $\left[-1.2 \mathrm{nN} \sigma^{7} / q^{7}\right.$, Eq. (17)] exceeds the vdW force calculated by the continuum approach for $q \approx \sigma$. It is also noted that the chemical force contribution to $F_{t s}$ should be strongly site dependent, i.e., the strong chemical attraction can only occur if front atom and sample atom can form a bond. Repulsive interaction is expected at any site whenever $q$ is small enough, though its magnitude may vary.

\section{STABILITY CRITERION FOR DYNAMIC AFM}

For attractive tip-sample forces, the CL usually jumps to a surface if the distance is less than a certain distance $d$ $=d_{\text {min }}$. This "jump-to-contact" (JTC) has to be avoided when trying to achieve atomic resolution because (a) an atomically sharp tip will get crushed if it is pierced uncontrolled into a surface and (b) in FM-AFM the oscillation of the CL would stop if it sticks to the surface. On the other hand, it is mandatory to get very close to the surface, since for achieving atomic resolution the force between the front atom and sample has to be a significant fraction of the total tip sample force.

The necessary condition for JTC is that the magnitude of the second derivative of the attractive tip-sample potential is greater than the spring constant of the CL: ${ }^{24}$

$$
\frac{\partial^{2} V_{t s}}{\partial d^{2}}>k
$$

However, this is not a sufficient condition. A second condition for JTC is that the net force acting on the CL has to be negative. This second condition is usually not emphasized because it is fulfilled automatically in static force microscopy. In dynamic force microscopy the net force exerted on the tip is $F_{t s}$ plus $F_{\text {spring }}$, the retracting force of the deflected CL. $F_{\text {spring }}=-k q^{\prime}$ and $q^{\prime}=-A_{0}$ when $F_{t s}$ is maximal, thus the second criterion for JTC to occur is

$$
k A_{0}+F_{t s}<0
$$

Typical operating parameters in FM-AFM are $A_{0}$ $\approx 10 \mathrm{~nm}$ and $k=20 \mathrm{~N} / \mathrm{m}$, thus $F_{t s}$ would need to exceed $-200 \mathrm{nN}-$ a value that could only happen with a very blunt tip and/or additional attractive forces (e.g., electrostatic).

\section{COMPARISON TO EXPERIMENT}

\section{A. Dependence of $\Delta f\left(A_{0}\right)$}

In order to measure $\Delta f\left(A_{0}\right)$, the closest distance between $\mathrm{CL}$ and sample $d$ has to be kept constant while varying the amplitude $A_{0}$. In praxi, this is a fairly difficult task because the sample has to be retracted from the CL by exactly the same amount the amplitude is increased. However, it is sensible to assume that when imaging a specific sample with a specific tip with atomic resolution with two different amplitudes, $d$ will be similar within fractions of a nm. Kitamura and Iwatsuki have done that experiment and found that the gradients of $\Delta f(d)$ are $10 \mathrm{~Hz} / \mathrm{nm}$ for $A_{0}=10 \mathrm{~nm}$ and 150 $\mathrm{Hz} / \mathrm{nm}$ for $A_{0}=1.5 \mathrm{~nm}$. $^{12}$ The exponent derived by that data is $\ln (150 / 10) / \ln (1.5 / 10)=-1.43$, which is in excellent agreement with $\Delta f \propto A_{0}^{-1.5}$ as predicted by Eq. (13).

\section{B. Dependence of $\Delta f(d)$}

In general, the forces between a CL and a sample are composed of capillary, van der Waals, electrostatic, magnetic, and short-range chemical interactions. For a meaningful comparison between experiment and theory, the experimental situation has to be well defined. Pure vdW interaction across vacuum is such a well-defined situation. ${ }^{2}$ In this case, $\Delta f(d)$ is determined by the geometry of the tip and the Hamaker constant of the tip-sample combination. Three examples of experimental $\Delta f(d)$ data where the vdW interaction is dominant are compared to the following calculations: (a) Bammerlin et al.: ${ }^{15} \mathrm{NaCl}$ (001) sample, silicon tip; (b1) Sugawara et al.: ${ }^{25} \mathrm{Si}$ (111) sample, silicon tips; and (b2) Giessibl: ${ }^{7} \mathrm{Si}(111)$ sample, silicon tip.

Since the $-\ln (-\Delta f)$ versus $\ln (d)$ plot of the data above fits very well to a straight line with a slope of 0.5 [Eq. (23)] for distances where vdW forces are dominant, a pyramidal tip model is used as a tip model. Inserting Eq. (26) into Eq. (16) yields

$$
\begin{aligned}
\gamma(d)= & -\frac{1}{\sqrt{2} \pi}\left\{\frac{2}{3} \tan ^{2}(\alpha / 2) A_{H} \frac{1}{\sqrt{d}}\right. \\
& \left.+12 E_{\text {bond }}\left(0.7 \frac{\sigma^{6}}{d^{6.5}}-0.5 \frac{\sigma^{12}}{d^{12.5}}\right)\right\} .
\end{aligned}
$$

Figure 2 shows a fit of this curve to the experimental data sets (a)-(c). On a $\ln (-\gamma) / \ln (d)$ plot, the curve is a straight line for $d \geqq 2 \sigma$. For $d \lesssim 2 \sigma$, a "dip", occurs since the attractive chemical forces cause a stronger decrease in $\gamma$. If $d$ becomes even smaller, repulsive forces strongly increase and $\gamma$ crosses zero and reaches positive values (the plot only covers $\gamma<0$ ).

\section{Silicon tip/NaCl (001) sample}

The diamond-shaped data points have been derived with $f_{0}=168 \mathrm{kHz}, k=30 \mathrm{~N} / \mathrm{m}$, and $A_{0}=13 \mathrm{~nm}$. The data can be fitted well by a straight line for most of the distance values with a fit parameter $\xi_{a}:=\tan ^{2}\left(\alpha_{a} / 2\right) A_{H, \mathrm{Si}-\mathrm{NaCl}}=2.1 \mathrm{aJ} . \mathrm{NaCl}$ is not expected to form strong chemical bonds with the $\mathrm{Si}$ tip, accordingly the dip is rather small before the frequency shift decreases again and crosses zero. 


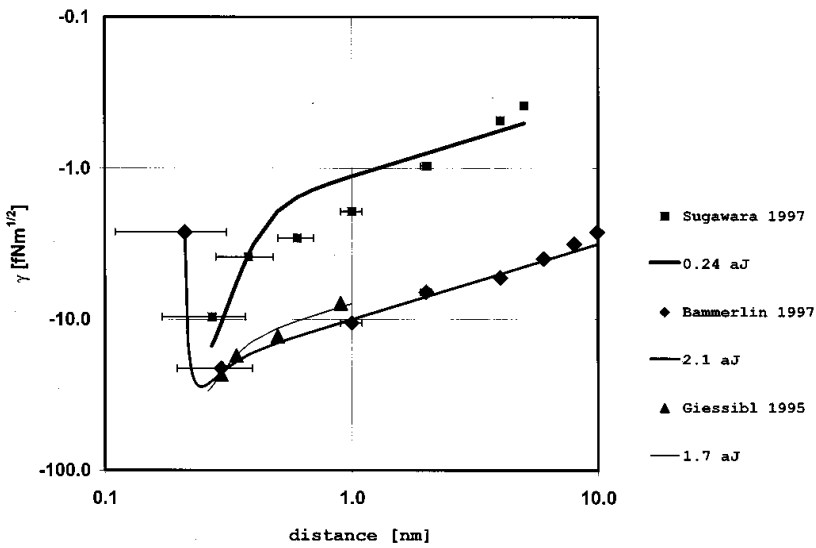

FIG. 2. Plot of the normalized frequency shift for three experimental data sets and fitted theoretical curves with $\xi:=\tan ^{2}(\alpha / 2) A_{H}$ as a fit parameter.

\section{Silicon tip/Si (111) sample}

The square-shaped data points have been derived with $f_{0}=172 \mathrm{kHz}, k=41 \mathrm{~N} / \mathrm{m}$, and $A_{0}=16 \mathrm{~nm}$ (values for $f_{0}, k$, $A_{0}$ : Ref. 26). With $\xi_{b}:=\tan ^{2}\left(\alpha_{b} / 2\right) A_{H, \mathrm{Si}-\mathrm{Si}}$ the fit corresponds to $\xi_{b 1}=0.24 \mathrm{aJ}$. The triangular data points are taken with a silicon tip imaging $\operatorname{Si}(111)$ with $f_{0}=114 \mathrm{kHz}, k$ $=17 \mathrm{~N} / \mathrm{m}$, and $A_{0}=6 \mathrm{~nm}$. Here, $\xi_{b 2}=1.7 \mathrm{aJ}$. Since tip and sample material were similar in both experiments, the large difference of $\gamma$ for $d \geqslant 2 \sigma$ can only be explained by sharper tips in case of (b1). With $A_{H, \mathrm{Si}-\mathrm{Si}}=0.1865$ aJ (Ref. 19) $\alpha_{b 1}$ $=97^{\circ}$, and $\alpha_{b 2}=143^{\circ}$.

\section{SUMMARY AND CONCLUSION}

(1) There are two nontrivial findings for $\Delta f\left(d, k, A_{0}, f_{0}, n\right)$ in the case of large amplitudes: (a) $\Delta f$ $\propto A_{0}^{-3 / 2}$ and (b) $\Delta f(d) \propto d^{-n+1 / 2}$ for tip-sample forces $F_{t s}$ $\propto q^{-n}$. These findings are confirmed by published experimental data.

(2) $\Delta f\left(d, k, A_{0}, f_{0}\right)$ can be condensed in a "normalized" frequency shift $\gamma(d):=\Delta f\left(d, k, A_{0}, f_{0}\right) k A_{0}^{3 / 2} / f_{0}$ such that different experimental results can be compared. The unit of $\gamma$ is $N \sqrt{m}$. Also, the introduction of $\gamma$ allows one to relate the experimental parameters to the forces that are acting between tip and sample:

$$
\gamma(d)=-\frac{1}{\sqrt{2} \pi} \sum_{n=1}^{\infty} \frac{C_{n} I_{1}(n)}{d^{n-1 / 2}}=\frac{\sqrt{d}}{\sqrt{2} \pi} \sum_{n=1}^{\infty} I_{1}(n) F_{t s}^{n}(d) .
$$

For our simple model for $F_{t s}$ [Eq. (26)] $\gamma$ is related to the force components by

$$
\begin{aligned}
\gamma(d)= & \frac{\sqrt{d}}{\sqrt{2} \pi}\left\{\pi F_{\mathrm{vdW}, m=2}(d)+0.7 F_{\text {LJ-att }}(d)\right. \\
& \left.+0.5 F_{\text {LJ-rep }}(d)\right\} .
\end{aligned}
$$

The $\Delta f(d)$ curves published in the literature show discontinuity for large positive frequency shifts. ${ }^{9}$ Using Eq. (31) shows that the repulsive forces on the front atom exceed several $\mathrm{nN}$, suggesting that the discontinuity is caused by nonreversible tip fracture.

(3) An extended "jump-to-contact" criterion has been established that explains why large amplitudes in dynamic AFM are required for obtaining atomic resolution.

Even though excellent tips for atomic-resolution AFM are now available commercially and scanning electron microscopy (SEM) images show extremely sharp conical tips with $\alpha \approx 30^{\circ}$ down to the resolution limit of the SEM, the geometry of the last few nm of the tip apex cannot be determined by SEM. Usually, the commercial tips point in the [001] direction. Since for $\mathrm{Si},\{111\}$ planes have the lowest surface energy per area there is hope that silicon tips pointing in a [001] direction could be prepared to be bound by four $\{111\}$ planes. $^{27}$ Careful analysis of $\gamma(d)$ could help to identify effective tip preparation techniques.

\section{ACKNOWLEDGMENTS}

I wish to thank Hartmut Bielefeldt, Stefan Hembacher, Hans Hilgenkamp, and Jochen Mannhart for discussions. This work was supported by BMBF Grant 13N6918.
*Electronic address: franz.giessibl@physik.uni-augsburg.de

${ }^{1}$ G. Binnig, C. F. Quate, and Ch. Gerber, Phys. Rev. Lett. 56, 930 (1986).

${ }^{2}$ Electrostatic and magnetic components can be eliminated by equalizing the tip-sample potential and choosing nonmagnetic tips; chemical forces have a very short range.

${ }^{3}$ T. R. Albrecht, P. Grütter, D. Horne, and D. Rugar, J. Appl. Phys. 69, 668 (1991).

${ }^{4}$ The oscillation is sustained by positive feedback: the deflection signal is fed back to the CL, some implementations actually control the magnitude of $A_{0}$, others just supply a constant driving amplitude to an actuator, which shakes the CL. For piezoresistive CL's, it is even possible to use the back action of the deflection sensor to both sense and drive the CL; see F. J. Giessibl and M. Tortonese, Appl. Phys. Lett. 70, 2529 (1997).

${ }^{5}$ This is in contrast to "amplitude modulation (AM) technique," the earliest non-contact technique where $f$ is set by an external oscillator, see Y. Martin, C. C. Williams, and H. K. Wickramasinghe, J. Appl. Phys. 61, 4723 (1987).
${ }^{6}$ Park Scientific Instruments, 1171 Borregas Ave, Sunnyvale, CA; Olaf Ohlsson, Nanosensors GmbH, Wetzlar, Germany.

${ }^{7}$ F. J. Giessibl, Science 260, 67 (1995).

${ }^{8}$ S. Kitamura and M. Iwatsuki, Jpn. J. Appl. Phys., Part 2 34, L145 (1995).

${ }^{9}$ P. Güthner, J. Vac. Sci. Technol. B 14, 2428 (1996).

${ }^{10}$ R. Lüthi, E. Meyer, M. Bammerlin, A. Baratoff, T. Lehmann, L. Howald, Ch. Gerber, and H.-J. Güntherodt, Z. Phys. B 100, 165 (1996).

${ }^{11}$ R. Erlandsson, L. Olsson, and P. Martensson, Phys. Rev. B 54, R8309 (1996).

${ }^{12}$ S. Kitamura and M. Iwatsuki, Jpn. J. Appl. Phys., Part 2 35, L668 (1996).

${ }^{13}$ Y. Sugawara, M. Ohta, H. Ueyama, and S. Morita, Science 270, 1646 (1995).

${ }^{14}$ J. Patrin (unpublished).

${ }^{15}$ M. Bammerlin, R. Lüthi, E. Meyer, A. Baratoff, J. Lü, M. Guggisberg, Ch. Gerber, L. Howald, H.-J. Güntherodt, Probe Microscopy 1, 3 (1997). 
${ }^{16}$ H. Goldstein, Classical Mechanics (Addison-Wesley, Reading, MA, 1980).

${ }^{17}$ Calculating $\Delta f$ for $F_{t s}(q)=F_{0} e^{-\alpha q}$ yields $\Delta f=\left(f_{0} / k A_{0}^{3 / 2}\right)$ $F_{0} e^{-\alpha q} 1 / \sqrt{2 \alpha \pi}$.

${ }^{18}$ E. Meyer and H. Heinzelmann, in Scanning Tunneling Microscopy II, edited by R. Wiesendanger and H. J. Güntherodt (Springer, Berlin, Heidelberg 1992), (1995 2nd ed.), p. 99; N. Burnham and R. J. Colton, in Scanning Tunneling Microscopy and Spectroscopy: Theory, Techniques, and Applications, edited by D. A. Bonnell (VCH, New York 1993); S. Ciraci, in Scanning Tunneling Microscopy III, edited by R. Wiesendanger and H. J. Güntherodt (Springer, Berlin, 1996), U. Landmann and W. D. Luedtke, ibid. p. 207; U. Hartmann, ibid. p. 293.

${ }^{19}$ R. Perez, M. C. Payne, I. Stich, and K. Terakura, Phys. Rev. Lett. 78, 678 (1997).

${ }^{20}$ J. Israelachvili, Intermolecular and Surface Forces (Academic Press, London, 1985).
${ }^{21}$ Pyramidal tips that are crushed (i.e., the apex is flat because it has been pressed against the sample with a large force) can be modeled by $A(h)=\chi(h+\Delta)^{2}=\chi\left(h^{2}+\Delta h+\Delta^{2}\right)$.

${ }^{22}$ C. Kittel, Introduction to Solid State Physics, (Wiley, New York, 1986), p. 55.

${ }^{23}$ F. H. Stillinger and T. A. Weber, Phys. Rev. B 31, 5262 (1985).

${ }^{24}$ N. Burnham and R. J. Colton, J. Vac. Sci. Technol. A 7, 2906 (1989).

${ }^{25}$ Y. Sugawara, H. Ueyama, T. Uchihashi, M. Ohta, Y. Yanase, T. Shigematsu, M. Suzuki, and S. Morita (unpublished).

${ }^{26}$ Y. Sugawara (private communication).

${ }^{27}$ Silicon pyramids bound by $\{111\}$ planes even form by selfassembly when growing silicon on $\mathrm{Si}(100)$ through a mask by molecular beam epitaxy: H. Baumgärtner, W. Hansch, F. Wittmann, and I. Eisele (Fig. 4.7), Current Topics in Crystal Growth Res. 2, 283 (1995). 